\title{
Abundant Lump-Type Solutions and Interaction Solutions for a Nonlinear (3+1) Dimensional Model
}

\author{
R. Sadat $\mathbb{D}^{1},{ }^{1}$ M. Kassem, ${ }^{1}$ and Wen-Xiu Ma $\mathbb{i D}^{2,3,4,5}$ \\ ${ }^{1}$ Department of Mathematics and Physics, Faculty of Engineering, Zagazig University, Egypt \\ ${ }^{2}$ Department of Mathematics and Statistics, University of South Florida, Tampa, FL 33620, USA \\ ${ }^{3}$ Department of Mathematics, King Abdulaziz University, Jeddah, Saudi Arabia \\ ${ }^{4}$ College of Mathematics and Systems Science, Shandong University of Science and Technology, Qingdao 266590, Shandong, China \\ ${ }^{5}$ International Institute for Symmetry Analysis and Mathematical Modelling, Department of Mathematical Sciences, \\ North-West University, Mafikeng Campus, Mmabatho 2735, South Africa
}

Correspondence should be addressed to R. Sadat; eng_rahmaelsadat@yahoo.com

Received 15 October 2018; Accepted 29 November 2018; Published 10 December 2018

Academic Editor: Qin Zhou

Copyright (C) 2018 R. Sadat et al. This is an open access article distributed under the Creative Commons Attribution License, which permits unrestricted use, distribution, and reproduction in any medium, provided the original work is properly cited.

\begin{abstract}
We explore dynamical features of lump solutions as diversion and propagation in the space. Through the Hirota bilinear method and the Cole-Hopf transformation, lump-type solutions and their interaction solutions with one- or two-stripe solutions have been generated for a generalized $(3+1)$ shallow water-like (SWL) equation, via symbolic computations associated with three different ansatzes. The analyticity and localization of the resulting solutions in the $(x, y, z$, and $t)$ space have been analyzed. Threedimensional plots and contour plots are made for some special cases of the solutions to illustrate physical motions and peak dynamics of lump soliton waves in higher dimensions. The study of lump-type solutions moderates the visuality of optics media and oceanography waves.
\end{abstract}

\section{Introduction}

It is very important to control the physical mechanisms of rough waves and interaction waves specially with lumptype waves. The significance of nonlinear waves of these types appears from natural disasters. Many physical phenomena need analytical approaches to classify the physical dynamics of nonlinear evaluation equations. The Darboux transformation (DT) and the Lie symmetry (LS) method [13] are efficient approaches to obtaining closed-form solutions. However, some problems occur in applying those methods, such as how to find Lax pairs in the DT method and how to carry out the back-substitution procedure in the LS method. There are also new types of closed-form solutions, for example, positions and complexions [4-8], and even new collision phenomena including fissions and fusions [914]. The Hirota bilinear method plays an influential role in discovering all the mentioned types of solutions to overcome a lot of analytic problems. Most studies apply the Hirota method to completely integrability nonlinear problems as in
$[10,15-23]$. We would like to demonstrate that the Hirota method can be used to explore various types of closed-form solutions: interaction solutions of lumps with solitons, kinks, line-solitons, resonance solutions, and one- or two-stripe solitons; and two classes of breather solutions (time periodic or space periodic solutions). Our analysis will show that those solutions can predicate the characteristics and physical significance of nonlinear problems.

Consider the following generalized $(3+1)$ SWL equation $[24,25]$ :

$$
u_{x x x y}+3 u_{x x} u_{y}+3 u_{x} u_{x y}-u_{y t}-u_{x z}=0
$$

There are a few studies on this equation. For example, Tian et al. in [26] generated a traveling wave solution via the tanh method. In 2010, Zayed [27] used the $G^{\prime} / G$ method to obtain some traveling wave solutions by reducing the independent variables using the linear D'lambert transformation.

In what follows, we investigate lump soliton solutions and their dynamics and the susceptibility of their interactions 
with other types of solutions using the Hirota method for (1). By using the singular manifold method (SMM) with twoterm truncated series, one derives the same ansatz in $[24,25]$

$$
u(x, y, t, z)=2\left(\ln (\psi(x, y, t, z))_{x} .\right.
$$

This is called the Cole-Hopf transformation, where $\psi$ is an auxiliary or test function that will be determined later. Starting by substituting (2) into (1), one gets

$$
\begin{aligned}
8 \psi_{x x x y} \psi_{x} \psi-4 \psi_{x x x} \psi_{x y} \psi+2 \psi_{x x x x} \psi_{y} \psi-12 \psi_{x x y} \psi_{x}{ }^{2} \\
-2 \psi_{x t} \psi_{y} \psi-2 \psi_{x y} \psi_{t} \psi-2 \psi_{y t} \psi_{x} \psi-2 \psi_{x x} \psi_{z} \psi \\
-4 \psi_{x z} \psi_{x} \psi+4 \psi_{x}{ }^{2} \psi_{z}+2 \psi_{x x z} \psi^{2}-2 \psi_{x x x x y} \psi^{2} \\
+2 \psi_{x y t} \psi^{2}-4 \psi_{x x x} \psi_{x} \psi_{y}+12 \psi_{x x} \psi_{x} \psi_{x y} \\
+4 \psi_{x} \psi_{t} \psi_{y}=0 .
\end{aligned}
$$

The transformation increases the nonlinearity but allows us to work with the test function. In [24], Zhang used Bell polynomial theories to generate lump-kink solutions, lumps with one-stripe solitons and lumps with two-stripe solitons for (1), but he supposed that $z=x$ to minimize the number of independent variables and so studied the equation in a $(2+1)$ dimensional domain.

\section{Lump Soliton Solutions}

To generate single-lump solutions, we suppose that

$$
\begin{aligned}
& \psi=\beta^{2}+\gamma^{2}+\alpha_{11}, \\
& \beta=\alpha_{1} x+\alpha_{2} y+\alpha_{3} t+\alpha_{4} z+\alpha_{5}, \\
& \gamma=\alpha_{6} x+\alpha_{7} y+\alpha_{8} t+\alpha_{9} z+\alpha_{10} .
\end{aligned}
$$

where $\alpha_{i}, i=1 \ldots 11$, are real unknowns that will be found subsequently. We carry out a direct substitution of (4) into (3) and gather the coefficients of the resulting polynomial in $x, y, t$, and $z$, to obtain a nonlinear algebraic system in $\alpha_{k}$. By solving this system of nonlinear algebraic equations with the aid of Maple, we acquire some sets of solutions for the parameters. Avoiding the redundancy, we surpass one studying case as follows:

$$
\begin{aligned}
\alpha_{1} & =\alpha_{1}, \\
\alpha_{2} & =\alpha_{2}, \\
\alpha_{3} & =-\frac{1}{\alpha_{6} \alpha_{11}\left(\alpha_{1} \alpha_{7}-\alpha_{2} \alpha_{6}\right)}\left(-\alpha_{1}^{2} \alpha_{7} \alpha_{8} \alpha_{11}\right. \\
& +\alpha_{2} \alpha_{1} \alpha_{6} \alpha_{8} \alpha_{11}+3 \alpha_{6}^{5} a_{7}+3 \alpha_{1}^{5} \alpha_{2}+6 \alpha_{1}^{3} \alpha_{6}^{2} \alpha_{2} \\
& \left.+3 \alpha_{1}^{4} \alpha_{6} \alpha_{7}+6 \alpha_{1}^{2} \alpha_{6}^{3} \alpha_{7}+3 \alpha_{6}^{4} \alpha_{1} \alpha_{2}\right), \\
\alpha_{4} & =\frac{1}{\alpha_{6} \alpha_{11}\left(\alpha_{1} \alpha_{7}-\alpha_{2} \alpha_{6}\right)}\left(3 \alpha_{1}^{4} \alpha_{2}^{2}+6 \alpha_{1}^{3} \alpha_{2} \alpha_{6} \alpha_{7}\right. \\
& +3 \alpha_{6}^{2} \alpha_{1}^{2} \alpha_{2}^{2}+3 \alpha_{6}^{2} \alpha_{1}^{2} \alpha_{7}^{2}-\alpha_{2} \alpha_{1} \alpha_{7} \alpha_{8} \alpha_{11}+6 \alpha_{6}^{3} \alpha_{1} \alpha_{2} \alpha_{7} \\
& \left.+\alpha_{2}^{2} \alpha_{11} \alpha_{6} \alpha_{8}+3 \alpha_{6}^{4} \alpha_{7}^{2}\right),
\end{aligned}
$$

$$
\begin{aligned}
& \alpha_{5}=\alpha_{5}, \\
& \alpha_{6}=\alpha_{6}, \\
& \alpha_{7}=\alpha_{7}, \\
& \alpha_{8}=\alpha_{8}, \\
& \alpha_{9}=\frac{\left(3 \alpha_{1}^{3} \alpha_{2}+3 \alpha_{1}^{2} \alpha_{6} \alpha_{7}+3 \alpha_{6}^{2} \alpha_{1} \alpha_{2}-\alpha_{7} \alpha_{8} \alpha_{11}+3 \alpha_{6}^{3} \alpha_{7}\right)}{\alpha_{6} \alpha_{11}}, \\
& \alpha_{10}=0, \\
& \alpha_{11}=\alpha_{11} .
\end{aligned}
$$

Using the aggregation equation (4), one can represent the auxiliary function as

$$
\begin{aligned}
\psi & =\left(\alpha_{1} x+\alpha_{2} y-\frac{1}{\alpha_{6} \alpha_{11}\left(\alpha_{1} \alpha_{7}-\alpha_{2} \alpha_{6}\right)}\left(-\alpha_{1}^{2} \alpha_{7} \alpha_{8} \alpha_{11}\right.\right. \\
& +\alpha_{2} \alpha_{1} \alpha_{6} \alpha_{8} \alpha_{11}+3 \alpha_{6}^{5} a_{7}+3 \alpha_{1}^{5} \alpha_{2}+6 \alpha_{1}^{3} \alpha_{6}^{2} \alpha_{2} \\
& \left.+3 \alpha_{1}^{4} \alpha_{6} \alpha_{7}+6 \alpha_{1}^{2} \alpha_{6}^{3} \alpha_{7}+3 \alpha_{6}^{4} \alpha_{1} \alpha_{2}\right) t \\
& +\frac{1}{\alpha_{6} \alpha_{11}\left(\alpha_{1} \alpha_{7}-\alpha_{2} \alpha_{6}\right)}\left(3 \alpha_{1}^{4} \alpha_{2}^{2}+6 \alpha_{1}^{3} \alpha_{2} \alpha_{6} \alpha_{7}\right. \\
& +3 \alpha_{6}^{2} \alpha_{1}^{2} \alpha_{2}^{2}+3 \alpha_{6}^{2} \alpha_{1}^{2} \alpha_{7}^{2}-\alpha_{2} \alpha_{1} \alpha_{7} \alpha_{8} \alpha_{11}+6 \alpha_{6}^{3} \alpha_{1} \alpha_{2} \alpha_{7} \\
& \left.\left.+\alpha_{2}^{2} \alpha_{11} \alpha_{6} \alpha_{8}+3 \alpha_{6}^{4} \alpha_{7}^{2}\right) z+\alpha_{5}\right)^{2}+\left(\alpha_{6} x+\alpha_{7} y+\alpha_{8} t\right. \\
& +\frac{\left(3 \alpha_{1}^{3} \alpha_{2}+3 \alpha_{1}^{2} \alpha_{6} \alpha_{7}+3 \alpha_{6}^{2} \alpha_{1} \alpha_{2}-\alpha_{7} \alpha_{8} \alpha_{11}+3 \alpha_{6}^{3} \alpha_{7}\right)}{\alpha_{6} \alpha_{11}} \\
& +z)^{2}+\alpha_{11},
\end{aligned}
$$

where

$$
\Delta=\left|\begin{array}{ll}
\alpha_{1} & \alpha_{2} \\
\alpha_{6} & \alpha_{7}
\end{array}\right| \neq 0, \quad \alpha_{6} \alpha_{11} \neq 0 .
$$

By using (2), the solution of (1) has the form

$$
u=4 \frac{\alpha_{1} \beta+\alpha_{6} \gamma}{\psi}
$$

Incorporating (6) and (5) into (8), one gets a class of lump solutions of (1) depicted in Figure 1.

\section{Interaction Solutions}

3.1. Lump Solitons with One-Stripe Waves. Suppose that the test function is a confederation of a quadratic function withan 


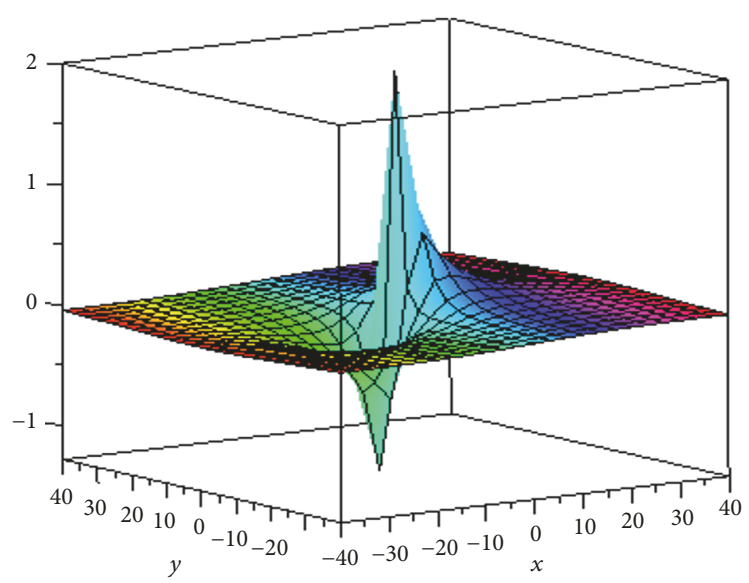

(a)

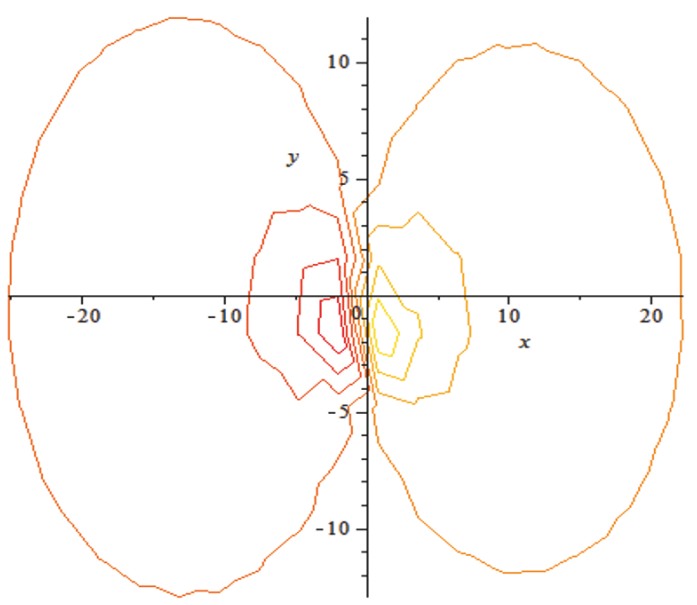

(d)

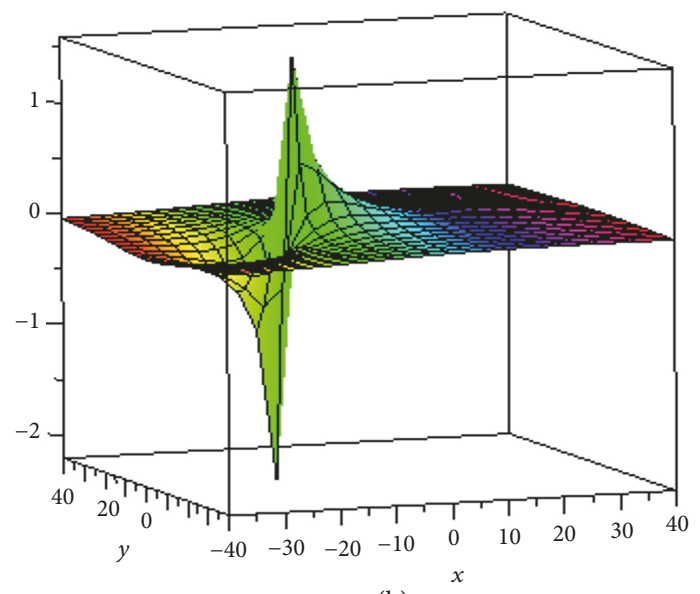

(b)

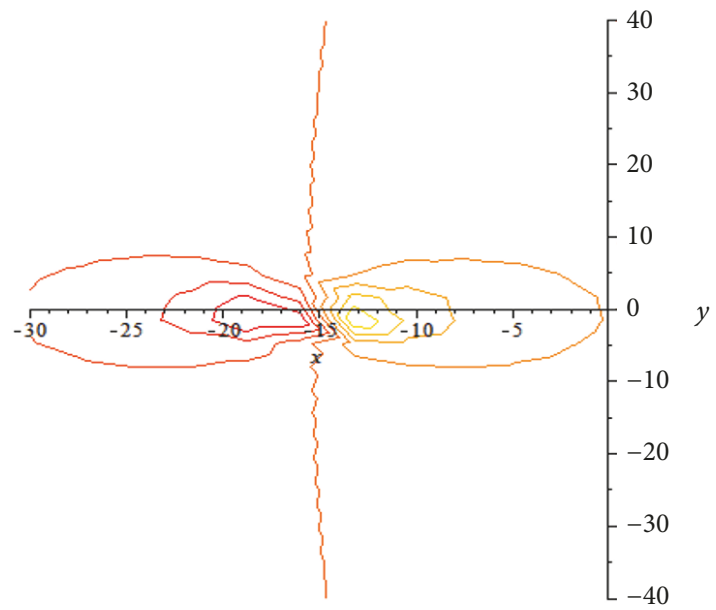

(e)

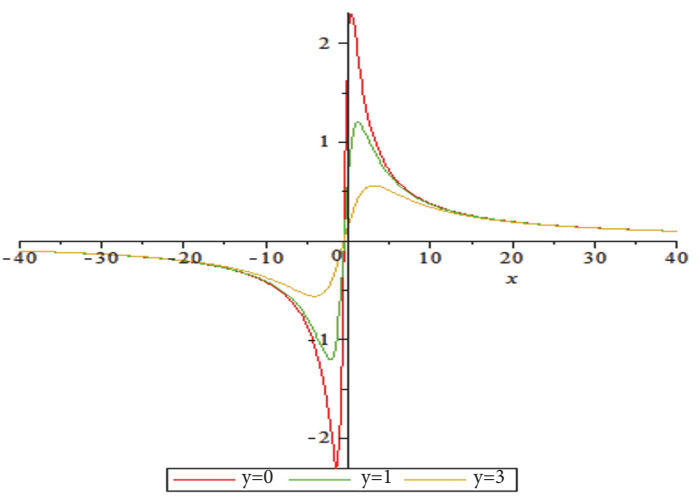

(f)

Figure 1: Proportion sight of the solution equation (8) with (6) and (5). For arbitrary constant values, $\alpha_{2}=\alpha_{1}=1, \alpha_{7}=-1, \alpha_{6}=1, \alpha_{10}=$ $0, \alpha_{11}=1$, and $\alpha_{8}=5$. (a-b) 3D plots for $t=0$, and 3, respectively. (d-e) Consistent contour plot of (a), (b). 2D plot present in (f) for various values of $y$.

exponential function as follows:

$$
\begin{aligned}
& \psi=\beta^{2}+\gamma^{2}+\alpha_{11}+\lambda, \\
& \beta=\alpha_{1} x+\alpha_{2} y+\alpha_{3} t+\alpha_{4} z+\alpha_{5}, \\
& \gamma=\alpha_{6} x+\alpha_{7} y+\alpha_{8} t+\alpha_{9} z+\alpha_{10}
\end{aligned}
$$

$$
\lambda=e^{k_{1} x+k_{2} y+k_{3} t+k_{4} z+k_{5}}
$$

where $\alpha_{i}, i=1 \ldots 11$ and $k_{j}, j=1 . .5$, are real unknown constants that will be determined subsequently. Using the ansatz in (2), 


$$
u=2 \frac{2 \alpha_{1} \beta+2 \alpha_{6} \gamma+k_{1} e^{k_{1} x+k_{2} y+k_{3} t+k_{4} z+k_{5}}}{\psi} .
$$

Inserting (9) into (3), gathering the coefficients of the resulting polynomial in $x, y, t$, and $z$, and equaling these coefficients to zero, we explore a complicated algebraic system on the unknown constants. We then solve the obtained system using Maple and snaffle the following assortment of solutions:

$$
\begin{gathered}
\alpha_{1}=\alpha_{1}, \\
\alpha_{2}=\frac{k_{2}\left(\alpha_{1}^{2}+\alpha_{6}^{2}\right)}{k_{1} \alpha_{1}}, \\
\alpha_{3}=3 k_{1}^{2} \alpha_{1}, \\
\alpha_{5}=\alpha_{5}, \\
\alpha_{6}=\alpha_{6} \\
\alpha_{7}=0 \\
\alpha_{8}=-3 \frac{k_{2}\left(\alpha_{1}^{2}\right)}{\alpha_{6}}, \\
\alpha_{9} \frac{k_{1} k_{2}\left(\alpha_{1}^{2}+\alpha_{6}^{2}\right)}{\alpha_{6}} \\
\alpha_{10}=0 \\
\alpha_{11}=\frac{\left(\alpha_{1}^{2}+\alpha_{6}^{2}\right)}{k_{1}^{2}} \\
k_{1}=k_{1}, \\
k_{2}=k_{2}, \\
k_{4}=0 \\
k_{5}, \\
k_{5} . \\
k_{1}, \\
k_{1}
\end{gathered}
$$

To avoid the singularity and promote the wave to localize in all directions, the following stipulation must be taken into consideration:

$$
k_{1} a_{1} a_{6} \neq 0 .
$$

Substituting (11) into (9), we obtain

$$
\begin{aligned}
\psi= & \left(\alpha_{1} x+\frac{k_{2}\left(\alpha_{1}^{2}+\alpha_{6}^{2}\right)}{k_{1} \alpha_{1}} y+3 k_{1}^{2} \alpha_{1} t+\alpha_{5}\right)^{2} \\
& +\left(\alpha_{6} x-3 \frac{k_{2}\left(\alpha_{1}^{2}\right)}{\alpha_{6}} t+\frac{3 k_{1} k_{2}\left(\alpha_{1}^{2}+\alpha_{6}^{2}\right)}{\alpha_{6}} z\right)^{2} \\
& +\frac{\left(\alpha_{1}^{2}+\alpha_{6}^{2}\right)}{k_{1}^{2}}+e^{k_{1} x+k_{2} y+k_{1}^{3} t+k_{5}}
\end{aligned}
$$

Introducing (13) into (10), we generate a class of interaction solutions with stripe soliton (solitary wave) solutions. The results have been plotted in Figure 2 for different values of times.

3.2. Lump Solitons with Tough Waves (Two-Stripe Solitons). We suppose that the new ansatz is a combination of a quadratic function and hyperbolic function as follows:

$$
\begin{aligned}
& \psi=\beta^{2}+\gamma^{2}+\alpha_{11}+\delta, \\
& \beta=\alpha_{1} x+\alpha_{2} y+\alpha_{3} t+\alpha_{4} z+\alpha_{5}, \\
& \gamma=\alpha_{6} x+\alpha_{7} y+\alpha_{8} t+\alpha_{9} z+\alpha_{10}, \\
& \delta=\cosh \left(k_{1} x+k_{2} y+k_{3} t+k_{4} z+k_{5}\right) .
\end{aligned}
$$

Substituting (17) into (2), we snaffle an assortment of solutions for (1) as follows:

$u$

$$
=2 \frac{2 \alpha_{1} \beta+2 \alpha_{6} \gamma+k_{1} \sinh \left(k_{1} x+k_{2} y+k_{3} t+k_{4} z+k_{5}\right)}{\psi} .
$$

More complicated calculations have been done using Maple, to acquire the unidentified constants. Substituting (14) into (3), equaling the coefficients of $x, y, t$, and $z$ to zero, and solving the resulting nonlinear algebraic system (up to 150 equations), we explore the following solution cases of the constant parameters. In each case, we do back substitution in (14).

$$
\begin{aligned}
& \alpha_{1}=0, \\
& \alpha_{2}=\frac{12\left(k_{2}^{2} \alpha_{4} \alpha_{6}^{2}\right)}{9 k_{2}^{2} \alpha_{6}^{2} k_{1}^{2}+4 \alpha_{4}^{2}}, \\
& \alpha_{3}=\frac{-k_{1} \alpha_{4}}{k_{2}}, \\
& \alpha_{4}=\alpha_{4}, \\
& \alpha_{5}=0, \\
& \alpha_{6}=\alpha_{6}, \\
& \alpha_{7}=\frac{\alpha_{6} k_{2}\left(4 \alpha_{4}^{2}-9 k_{2}^{2} \alpha_{6}^{2} k_{1}^{2}\right)}{k_{1}\left(4 \alpha_{4}^{2}+9 k_{2}^{2} \alpha_{6}^{2} k_{1}^{2}\right)}, \\
& \alpha_{8}=\frac{3\left(k_{1}^{2} \alpha_{6}\right)}{2}, \\
& \alpha_{9}=\frac{3\left(k_{1} k_{2} \alpha_{6}\right)}{2}, \\
& \alpha_{10}=0, \\
& \alpha_{11}=\frac{9 k_{2}^{2} \alpha_{6}^{2} k_{1}^{2}+4 k_{1}^{4} \alpha_{4}^{2}+16 \alpha_{4}^{2} \alpha_{6}^{2}-36 \alpha_{6}^{2} k_{1}^{2} k_{2}^{2}}{16 \alpha_{4}^{2} \alpha_{6}^{2} k_{1}^{2}},
\end{aligned}
$$




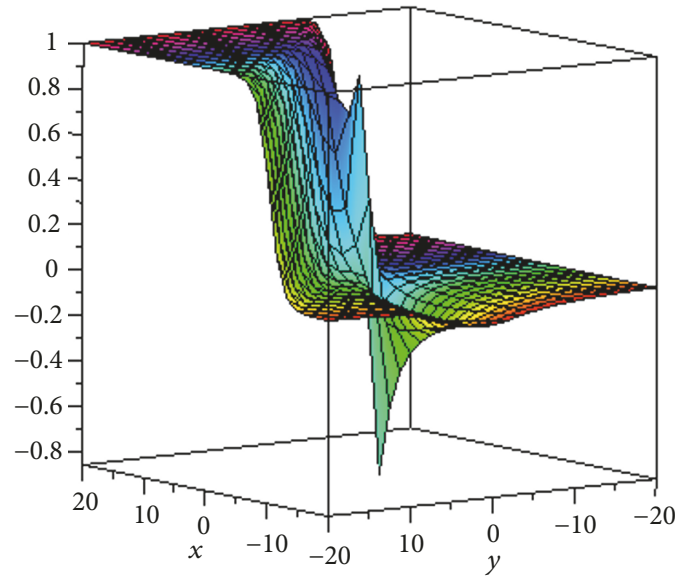

(a)

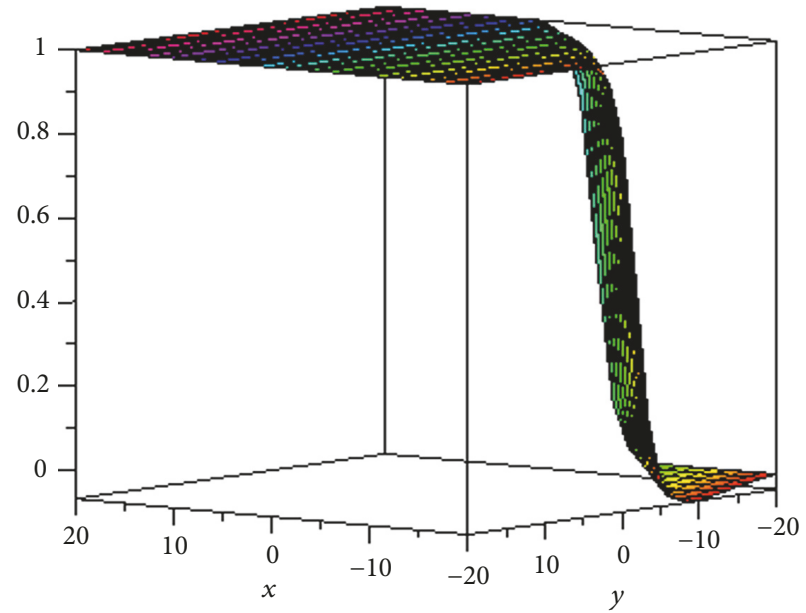

(c)

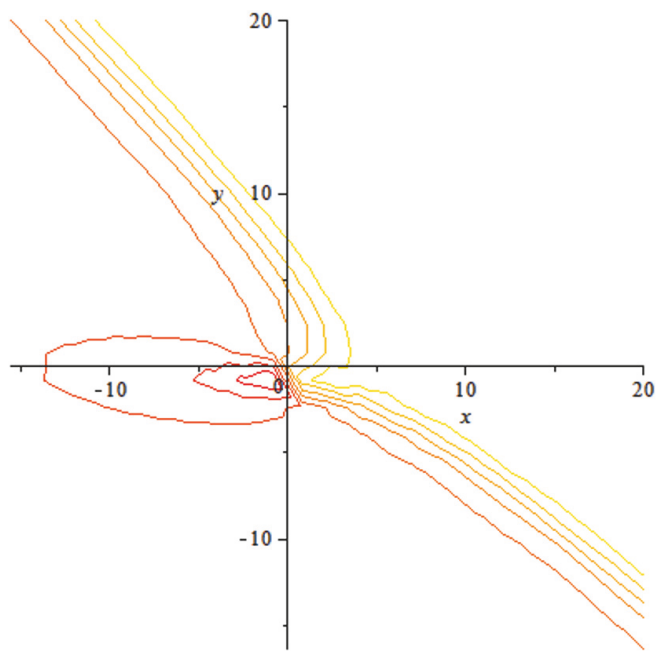

(f)

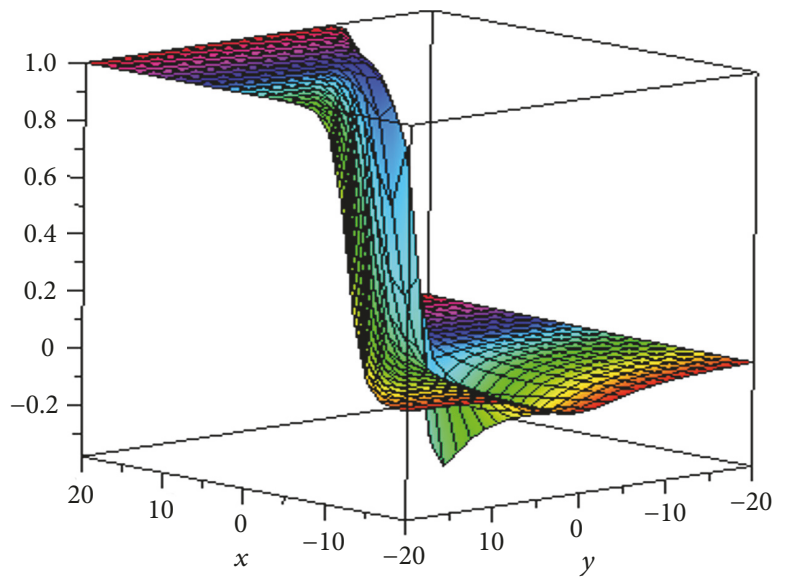

(b)

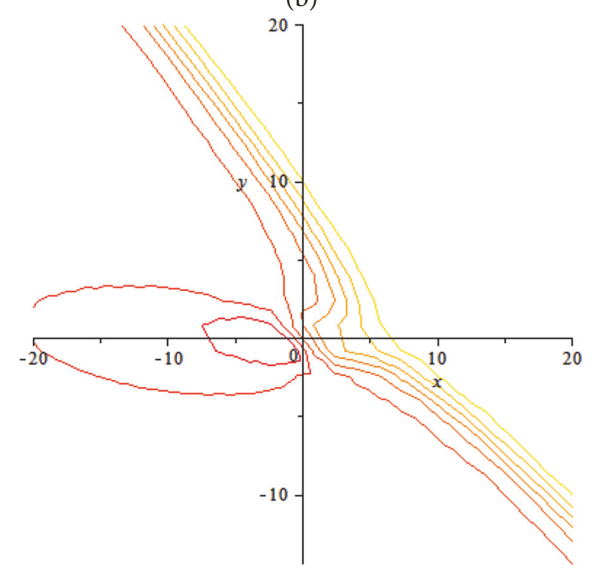

(e)

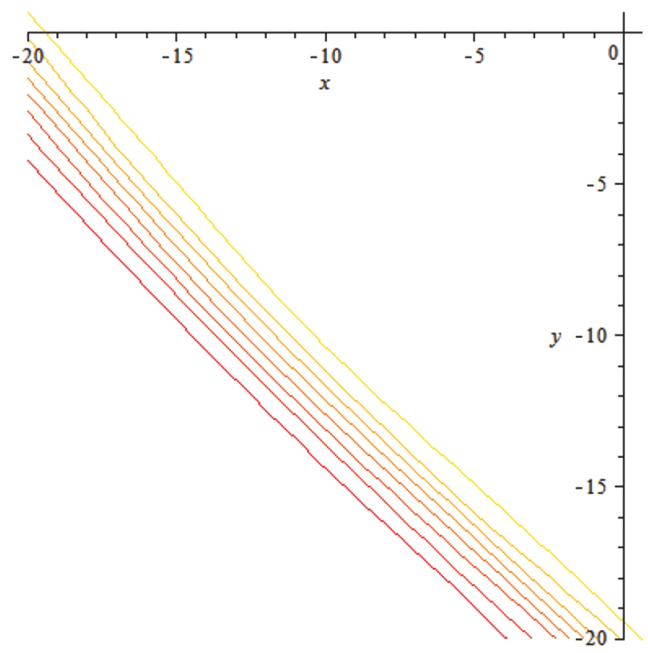

(g)

Figure 2: Proportion scene of the solution equation (10) with (13) and (11) for the values of arbitrary constants is $\alpha_{1}=1, \alpha_{4}=0, \alpha_{6}=3, \alpha_{5}=$ $1, \alpha_{10}=0$, and $k_{1}=k_{2}=k_{5}=1$. (a-c) represent 3D plots for (10) at $t=0,2$, and 30, $z=0$, respectively. (e-g) Consistent contour plot of (a, b, c), respectively. 


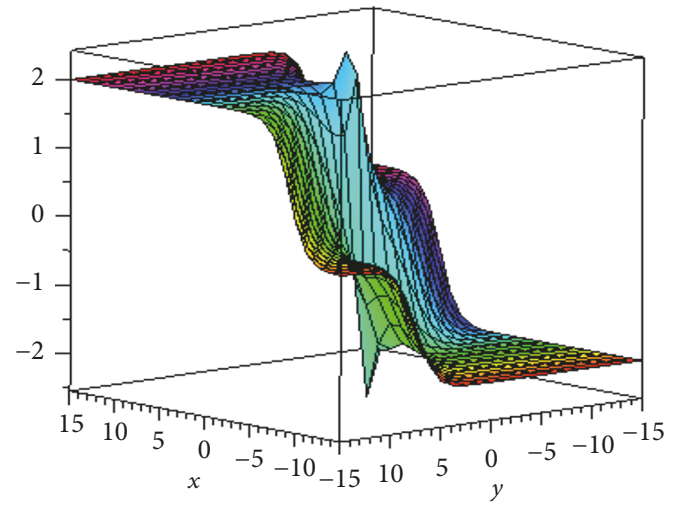

(a)

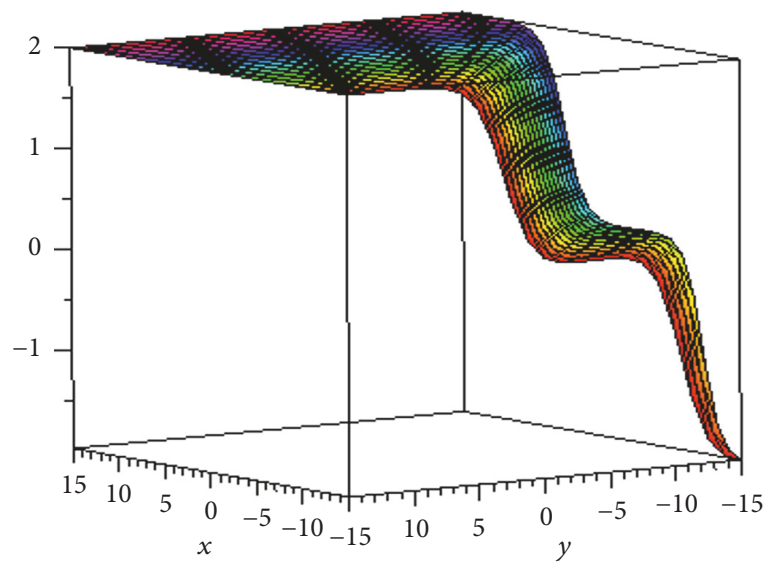

(c)

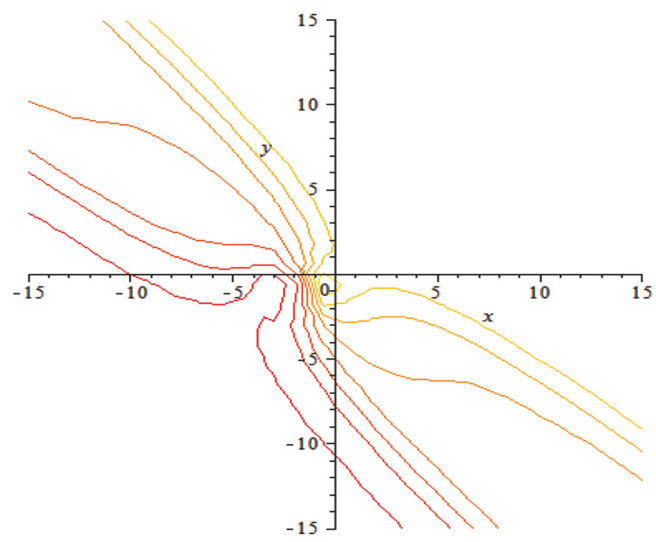

(f)

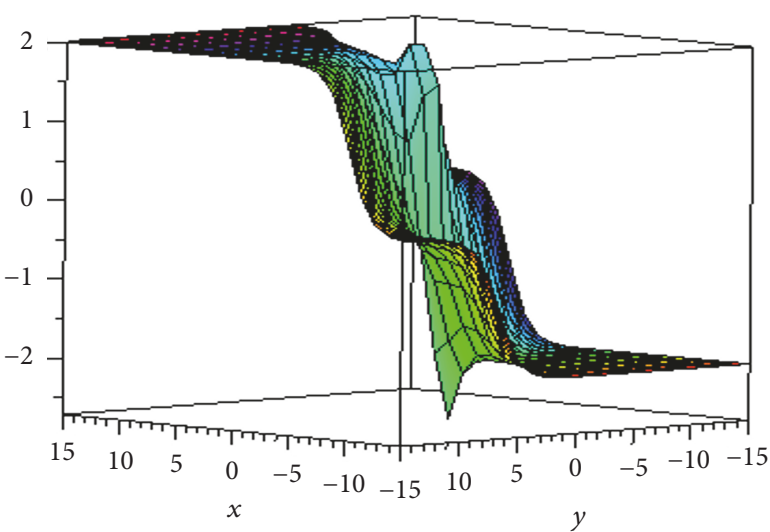

(b)

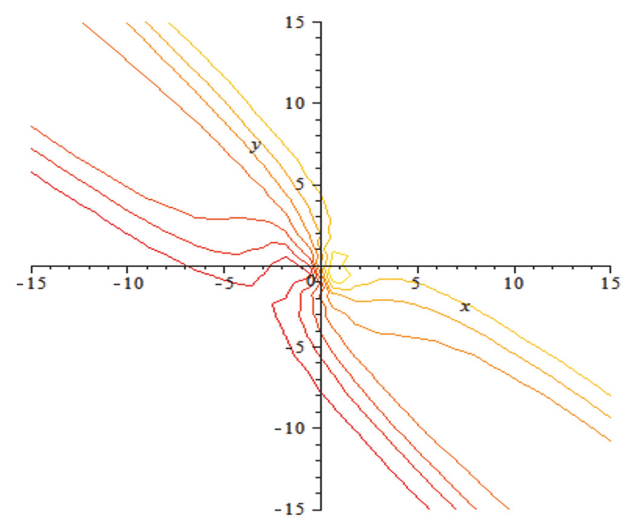

(e)

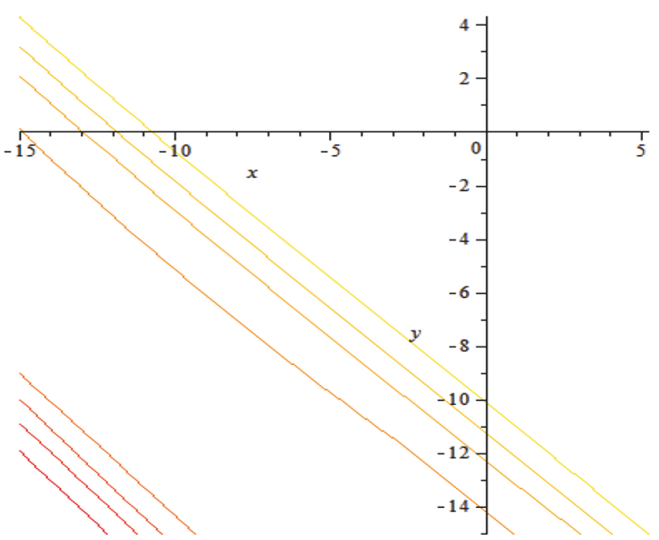

(g)

FIGURE 3: Proportion scenes of the solution equation (15) with (16) and (17) for the values of arbitrary constants are for $\alpha_{2}=-1, \alpha_{6}=1, \alpha_{7}=$ $2, \alpha_{5}=1, \alpha_{10}=0, \alpha_{8}=1, \alpha_{11}=1, k_{1}=1, k_{2}=1, k_{5}=1$. (a), (b), and (c) represent 3D plots for (10) at $t=0,5,18$ and $z=0$, respectively. (e), (f), and (g) Consistent contour plot of (a), (b), and (c).

$$
\begin{aligned}
& k_{2}=k_{2}, \\
& k_{3}=k_{1}^{3}, \\
& k_{4}=0, \\
& k_{5}=k_{5} .
\end{aligned}
$$

Substituting (16) into (14), we obtain

$$
\begin{aligned}
\psi & =\left(\alpha_{1} x+\frac{12\left(k_{2}^{2} \alpha_{4} \alpha_{6}^{2}\right)}{9 k_{2}^{2} \alpha_{6}^{2} k_{1}^{2}+4 \alpha_{4}^{2}} y-\frac{k_{1} \alpha_{4}}{k_{2}} t+\alpha_{4} z\right)^{2} \\
& +\left(\alpha_{6} x+\frac{\alpha_{6} k_{2}\left(4 \alpha_{4}^{2}-9 k_{2}^{2} \alpha_{6}^{2} k_{1}^{2}\right)}{k_{1}\left(4 \alpha_{4}^{2}+9 k_{2}^{2} \alpha_{6}^{2} k_{1}^{2}\right)} y+\frac{3\left(k_{1}^{2} \alpha_{6}\right)}{2} t\right.
\end{aligned}
$$




$$
\begin{aligned}
& \left.+\frac{3\left(k_{1} k_{2} \alpha_{6}\right)}{2} z\right)^{2} \\
& +\frac{9 k_{2}^{2} \alpha_{6}^{2} k_{1}^{2}+4 k_{1}^{4} \alpha_{4}^{2}+16 \alpha_{4}^{2} \alpha_{6}^{2}-36 \alpha_{6}^{2} k_{1}^{2} k_{2}^{2}}{16 \alpha_{4}^{2} \alpha_{6}^{2} k_{1}^{2}} \\
& +\cosh \left(k_{1} x+k_{2} y+k_{1}^{3} t+k_{5}\right)
\end{aligned}
$$

Through the same procedure, we get a class of solutions of (1) and plot a special solution in Figure 3.

\section{Conclusions}

Starting from the Cole-Hopf transformation, investigated in the SMM with a two-term truncated series, we derived novel lump solitons, lump-kinks, interacted lumps with onestripe solitons or kinks, and interacted lumps with two-stripe solitons or kink waves, after some complicated calculations using the Maple software. The presented three-dimensional plots of the interaction solutions show that the lump solitons are coalesced or spliced up by the stripe solitons. To the best of our knowledge, those types of solutions for (1) are presented for the first time. Our solutions are localized in the four dimensional space $(x, y, z$, and $t)$, but in [24], the authors assumed that $z=x$ and generated only one lump solution.

\section{Data Availability}

The data used to support the findings of this study are available from the corresponding author upon request.

\section{Conflicts of Interest}

The authors declare that they have no conflicts of interest.

\section{References}

[1] R. Sadat and A. Halim, "Explicit Solutions of a Generalized Hirota-Satsuma Equation Using Darboux Transformation," International Journal of Modern Mathematical Sciences, vol. 14, no. 3, pp. 325-334, 2016.

[2] R. Sadat and A. Halim, "New Soliton Solutions for the Kadomtsev-Petviashvili equation Using Darboux Transformation," International Journal of Modern Mathematical Sciences, vol. 15, pp. 112-122, 2017.

[3] R. Sadat and M. Kassem, "Explicit Solutions for the (2+1)Dimensional Jaulent-Miodek Equation Using the Integrating Factors Method in an Unbounded Domain," Mathematical \& Computational Applications, vol. 23, no. 1, Paper No. 15, 9 pages, 2018.

[4] Y. Zhou, Lump, Complexiton and Algebro-Geometric SOLutions to SOLiton Equations, 2017.

[5] P. G. Varlashkin and E. F. Zganjar, "Shifts of annihilation radiation energy from the electron rest mass energy for positons annihilating in copper and sodium," Nuclear Physics A, vol. 130, no. 1, pp. 182-194, 1969.
[6] W. Liu and Y. Zhang, "Resonant multiple wave solutions, complexiton solutions and rogue waves of a generalized $(3+1)$ dimensional nonlinear wave in liquid with gas bubbles," Waves in Random and Complex Media, pp. 1-11, 2018.

[7] Z. Sun, Y. Gao, X. Yu, W. Liu, and Y. Liu, "Bound vector solitons and soliton complexes for the coupled nonlinear Schrödinger equations," Physical Review E: Statistical, Nonlinear, and Soft Matter Physics, vol. 80, no. 6, 2009.

[8] B. Nawaz, S. T. Rizvi, K. Ali, and M. Younis, "Optical soliton for perturbed nonlinear fractional Schrödinger equation by extended trial function method," Optical and Quantum Electronics, vol. 50, no. 5, 2018.

[9] S. Wang, X.-Y. Tang, and S.-Y. Lou, "Soliton fission and fusion: Burgers equation and Sharma-Tasso-Olver equation," Chaos, Solitons \& Fractals, vol. 21, no. 1, pp. 231-239, 2004.

[10] Y. Zhou, S. Manukure, and W. X. Ma, "Lump and lump-soliton solutions to the Hirota-Satsuma-Ito equation," Communications in Nonlinear Science and Numerical Simulation, vol. 68, pp. 56-62, 2019.

[11] Z. Ma, J. Chen, and J. Fei, "Lump and line soliton pairs to a (2+1)-dimensional integrable KadomtsevPetviashvili equation," Computers \& Mathematics with Applications, 2018.

[12] X. Liu, H. Triki, Q. Zhou, W. Liu, and A. Biswas, "Analytic study on interactions between periodic solitons with controllable parameters," Nonlinear Dynamics, vol. 94, no. 1, pp. 703-709, 2018.

[13] C. Yang, W. Liu, Q. Zhou, D. Mihalache, and B. A. Malomed, "One-soliton shaping and two-soliton interaction in the fifthorder variable-coefficient nonlinear Schrödinger equation," Nonlinear Dynamics, pp. 1-12, 2018.

[14] K. Ali, S. T. R. Rizvi, S. Ahmad, S. Bashir, and M. Younis, "Bell and kink type soliton solutions in birefringent nano-fibers," Optik - International Journal for Light and Electron Optics, vol. 142, pp. 327-333, 2017.

[15] S. Batwa and W.-X. Ma, "A study of lump-type and interaction solutions to a (3+1)-dimensional Jimbo-Miwa-like equation," Computers \& Mathematics with Applications. An International Journal, vol. 76, no. 7, pp. 1576-1582, 2018.

[16] X. Yong, W.-X. Ma, Y. Huang, and Y. Liu, "Lump solutions to the Kadomtsev-Petviashvili I equation with a self-consistent source," Computers \& Mathematics with Applications, 2018.

[17] W.-X. Ma, "Lump solutions to the Kadomtsev-Petviashvili equation," Physics Letters A, vol. 379, no. 36, Article ID 23311, pp. 1975-1978, 2015.

[18] L. Cheng and Y. Zhang, "Lump-type solutions for the (4+ 1)-dimensional Fokas equation via symbolic computations," Modern Physics Letters B. Condensed Matter Physics, Statistical Physics, Applied Physics, vol. 31, no. 25, 1750224, 9 pages, 2017.

[19] J. Pu and H. Hu, "Construction of lump soliton and mixed lump stripe solutions of (3+1)-dimensional soliton equation," Applied Mathematics Letters, vol. 85, pp. 77-81, 2018.

[20] J. Yang, W. Ma, and Z. Qin, "Lump and lump-soliton solutions to the (2+1)-dimensional Ito equation," Analysis and Mathematical Physics, vol. 1, pp. 1-10, 2017.

[21] W. Tan and Z. Dai, "Spatiotemporal dynamics of lump solution to the (1+1)-dimensional Benjamin-Ono equation," Nonlinear Dynamics, vol. 89, no. 4, pp. 2723-2728, 2017.

[22] Y. Zhang, C. Yang, W. Yu, M. Mirzazadeh, Q. Zhou, and W. Liu, "Interactions of vector anti-dark solitons for the coupled nonlinear Schrödinger equation in inhomogeneous fibers," Nonlinear Dynamics, vol. 94, no. 2, pp. 1351-1360, 2018. 
[23] M. R. Ali and A. R. Hadhoud, "Hybrid Orthonormal Bernstein and Block-Pulse functions wavelet scheme for solving the $2 \mathrm{D}$ Bratu problem," Results in Physics, 2018.

[24] Y. Zhang, H. Dong, X. Zhang, and H. Yang, "Rational solutions and lump solutions to the generalized $(3+1)$-dimensional Shallow Water-like equation," Computers \& Mathematics with Applications, vol. 73, no. 2, pp. 246-252, 2017.

[25] Y.-N. Tang, W.-X. Ma, and W. Xu, "Grammian and Pfaffian solutions as well as Pfaffianization for a $(3+1)$-dimensional generalized shallow water equation," Chinese Physics B, vol. 21, no. 7, 2012.

[26] B. Tian and Y.-T. Gao, "Beyond travelling waves: A new algorithm for solving nonlinear evolution equations," Computer Physics Communications, vol. 95, no. 2-3, pp. 139-142, 1996.

[27] E. Zayed, "travelling wave solutions for higher dimensional nonlinear evaluation equations using G'G expansion method," Journal of Applied Mathematics \& Informatics, vol. 28, no. 1, 2, pp. 383-395, 2010. 


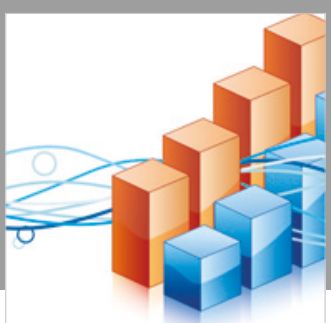

Advances in

Operations Research

\section{-n-m}
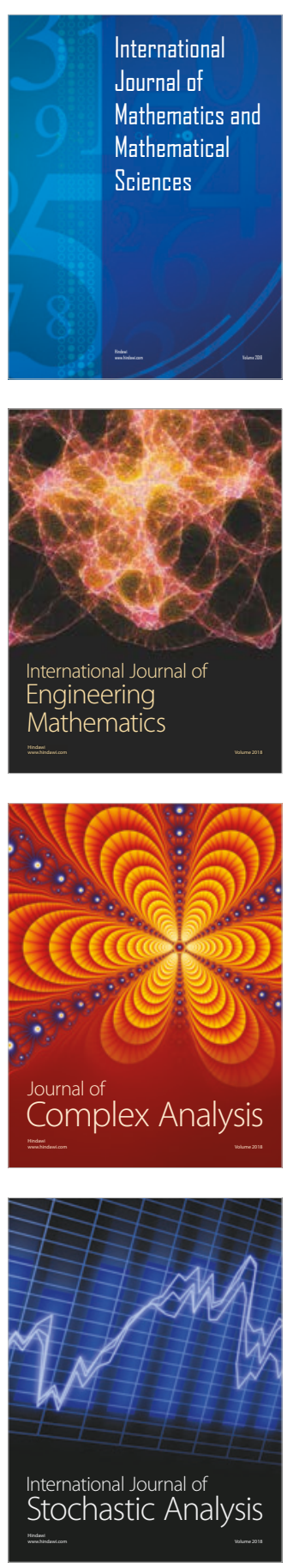
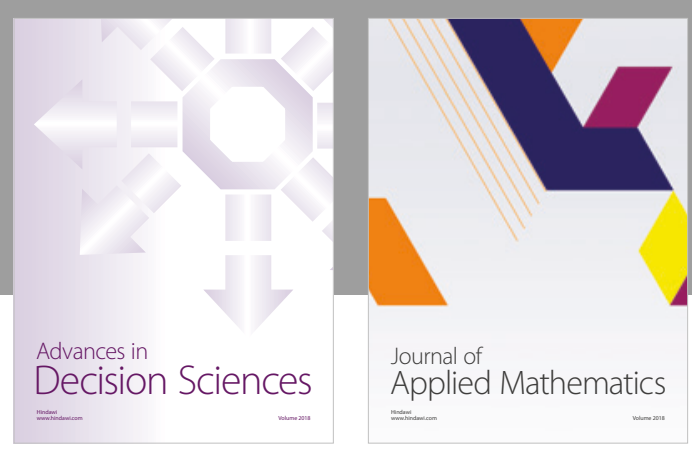

Journal of

Applied Mathematics
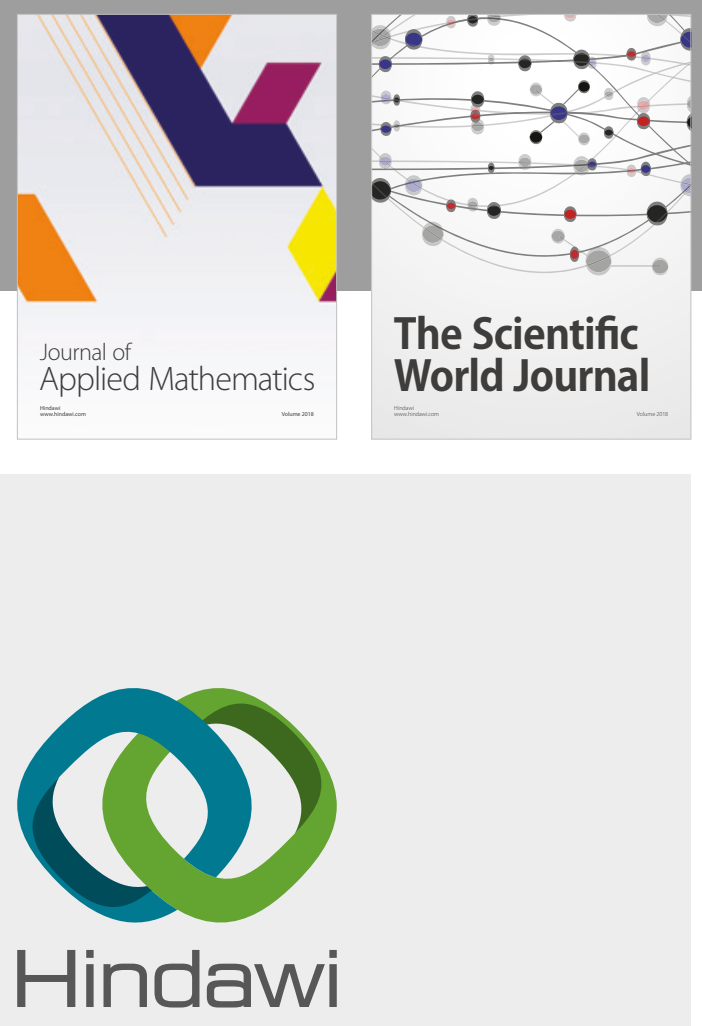

Submit your manuscripts at

www.hindawi.com

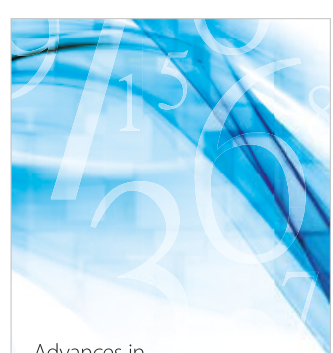

Advances in
Numerical Analysis
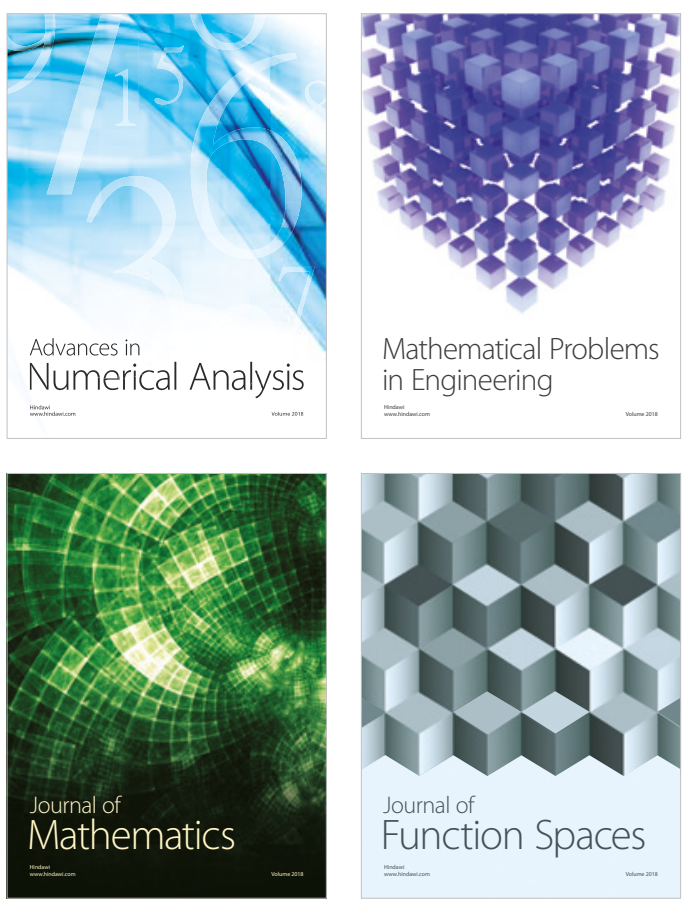

Mathematical Problems in Engineering

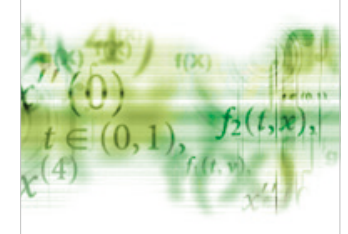

International Journal of

Differential Equations

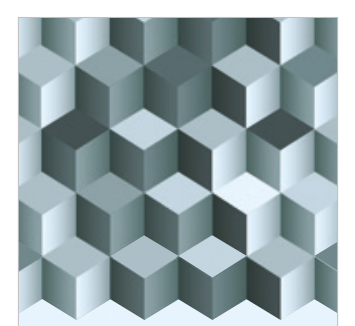

Journal of

Function Spaces

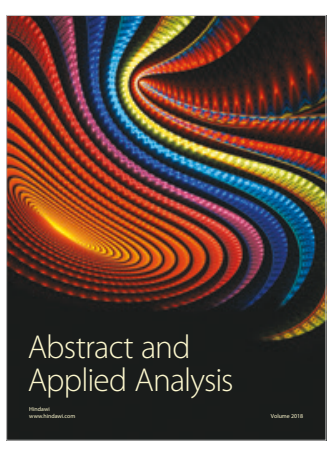

The Scientific

World Journal

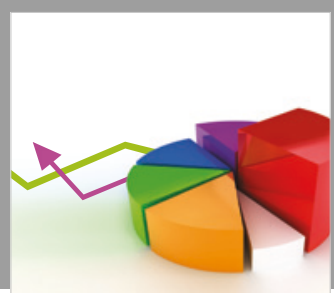

Journal of

Probability and Statistics
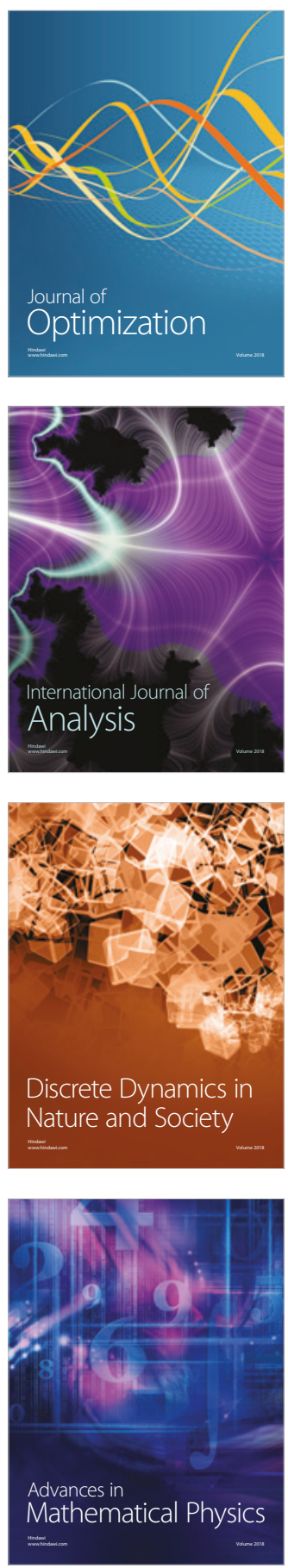